\title{
Approaches towards Structures of $Y$ Receptors, Examples of Human G-Protein Coupled Receptors, by Solution NMR
}

\author{
Reto Walser and Oliver Zerbe*
}

\begin{abstract}
Despite recent advances no solution structure for a true G-protein coupled receptor (GPCR) is available today due to biochemical and spectroscopic problems. Herein we review our attempts to obtain assignments of GPCRs based on fragments comprising 2-3 transmembrane helices. The fragments are expressed in a heterologous system, and studied in detergent micelles using solution NMR spectroscopy. We report on the status of assignments of fragments from the Y4 receptor, a human GPCR. Assignments for the majority of the backbone resonances are available as well as sidechain assignments for the first two TM helices. Residues of TM4 are largely invisible. We review technical issues in preparing these samples and in the data analysis. In addition we developed an approach in which we have grafted the extracellular loops of the Y1 receptor onto a beta-barrel scaffold derived from the $E$. coli outer membrane protein OmpA. We could demonstrate that all loops can be successfully transferred, and that the resulting protein can be successfully refolded. The system is capable of recognizing the ligands from the neuropeptide $Y$ family.
\end{abstract}

Keywords: GPCR $\cdot$ Grafting $\cdot$ OmpA $\cdot$ Y receptors

\section{Introduction}

The structural biology of G-protein coupled receptors has experienced dynamic progress during the last decade.[1] After the seminal paper from Palczewski on the structure of bovine rhodopsin in $2000^{[2]}$ a number of different GPCR structures have been solved over the last years, several in both the active and inactive states. This has tremendously improved our understanding of the activation process of these receptors and their underlying structural adaptations. Recently the first reports on structures of the complex of a ligand, its GPCR, and the cognate G-protein appeared. ${ }^{[3]}$ All these structures were solved using X-ray crystallography and, except for the rhodopsin structures, all GPCRs required stabilizing

\footnotetext{
${ }^{\star}$ Correspondence: Prof. Dr. O. Zerbe

University of Zurich

Institute of Organic Chemistry

Winterthurerstrasse 190

$\mathrm{CH}-8057$ Zürich

Tel.: +4144635 4263

Fax: +41446356882

E-mail: oliver.zerbe@oci.uzh.ch
}

mutations to enable crystallization and to render these receptors sufficiently thermostable for structural studies.

None of the high-resolution structures of GPCRs were determined using solution NMR studies so far. The structures of sensory rhodopsin, ${ }^{[4,5]}$ a 7 TM integral membrane protein with a topology similar to that of a GPCR, and for proteorhodopsin ${ }^{[6]}$ were recently determined in detergent micelles. Although both proteins are not coupled to a $\mathrm{G}$ protein, they also represent heptahelical membrane proteins with architectures very similar to true GPCRs. Both structures are well defined, and are considered to present structures comparable in quality to those derived from X-ray data. These milestone achievements indicate that high-quality NMR structures of heptahelical receptors are possible, provided that the system under study is sufficiently well behaved.[7] In addition sophisticated solid-state NMR was used to follow structural adaptations during activation. $\left.{ }^{8}\right]$

Despite these significant achievements, no NMR structures of true GPCRs are presently available, mainly due to a number of technical difficulties. Expression and purification of these membrane proteins under conditions that retain activity is a difficult issue. Another serious problem is the identification of sample conditions, under which the protein is sufficiently stable to allow recording all the required spectra at elevated temperature and with a spectral quality that permits assignment of the spectra. This issue is complicated by the fact that normally only a small amount of protein is available impeding screening of a larger set of conditions. The perspective of using NMR to investigate dynamics of GPCRs - information that is difficult to obtain from crystallographic studies merits major investments in methods development.

To bypass some of the technical difficulties when studying entire G-protein coupled receptors by solution NMR we are following a divide-and-conquer approach. Two approaches were followed by us, schematically depicted in Fig. 1. In the first we try to dissect the entire receptors into a set of smaller pieces, in the following referred to as fragments. These fragments are studied individually to learn about their conformational preferences: In particular, we are interested to see how stabilizing interactions with other parts of the protein result in conformational changes in the transmembrane (TM) helices. In addition these fragments allow us to study early events in folding of these membrane proteins since some of the long-range interactions with other parts of the receptor, e.g. interhelical contacts, are missing and hence they may provide model systems for the nascent polypeptide chain. Secondly, we hope to obtain chemical shift information that will be helpful when investigating the entire receptor. Finally, we hope to improve our understanding of lipid-protein interactions such that we will be able to select the best membrane-mimicking environment for the entire receptor later on. To be honest: The study of fragments is heavily under debate since we are investi- 
gating non-physiological systems. Part of our justification is based on the two-state membrane-protein folding model for helical membrane proteins that was developed by Popot and Engelman. ${ }^{[9]}$ In this model largely unfolded protein segments partition into the water-membrane interface during which they adopt secondary structure (coupled folding-partitioning). The helices then fully insert and diffuse in the membrane until (productive) tertiary contacts are formed. The important difference to most globular proteins is that fragments form secondary structure on their own! The fragmentation approach has therefore been used previously by other groups to study interesting domains of GPCRs. The design of so-called 'split receptors' of rhodopsin $^{[10]}$ and the $S$. cerevisiae $\alpha$-factor receptor Ste2 $\mathrm{p}^{[11]}$ has shown that receptor fragments can complement each other, restoring the functionality of the full-length receptor. Furthermore, the finding that fragments derived from the cytoplasmatic face of rhodopsin were able to competitively inhibit the interaction between rhodopsin and its G-protein transducin ${ }^{[12]}$ encouraged the first structure determination of such a fragment by NMR. [13]

Numerous studies by us during the last years have indicated that fragments corresponding to one or more TM helices do form stable secondary structure. ${ }^{[14-18]} \mathrm{We}$ have learned during those studies that the stability very much depends on the presence of polar residues in central regions of the TM helices. The exact structure of the TM helices in addition depends on tertiary contacts, and those may be missing in the segment. Therefore larger proteins comprising several TM helices inherently offer the possibility of stabilization of secondary structure via inter-helical contacts.

While this approach may offer some insight into the conformational preferences of TM helices it is unlikely to be able to report on the loops that connect the TM helices. In the GPCR structures published over the last 12 years, loops are sometimes less well defined. Class A GPCRs also possess an extracellular $\mathrm{N}$-terminal domain that usually is unfolded. Large ligands such as peptide hormones are believed to form contacts with the loops. To get some insight into ligand interactions with the loops we have transplanted the loops onto a beta barrel scaffold. ${ }^{[19,20]}$ Compared to the natural scaffold of a 7TM-bundle, these proteins offer the advantage that they can be produced in E. coli with high yields and are often easily purified. The importance of a rigid scaffold that will orient the three extracellular loops of the GPCR in one direction in space has been highlighted in previous studies of individual GPCR extracellular loops![21] Using a topological analysis of the published GPCR crystal structures

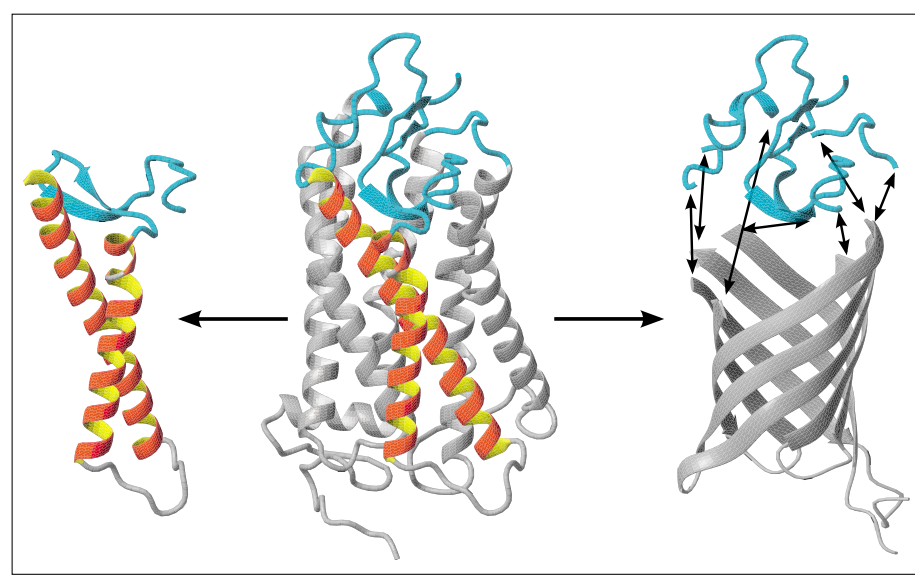

Fig. 1. Scheme highlighting the strategies for obtaining information on $\mathrm{Y}$ receptors developed in our laboratory. This involves the study of fragments (left) or the grafting of loops onto a rigid scaffold (right).

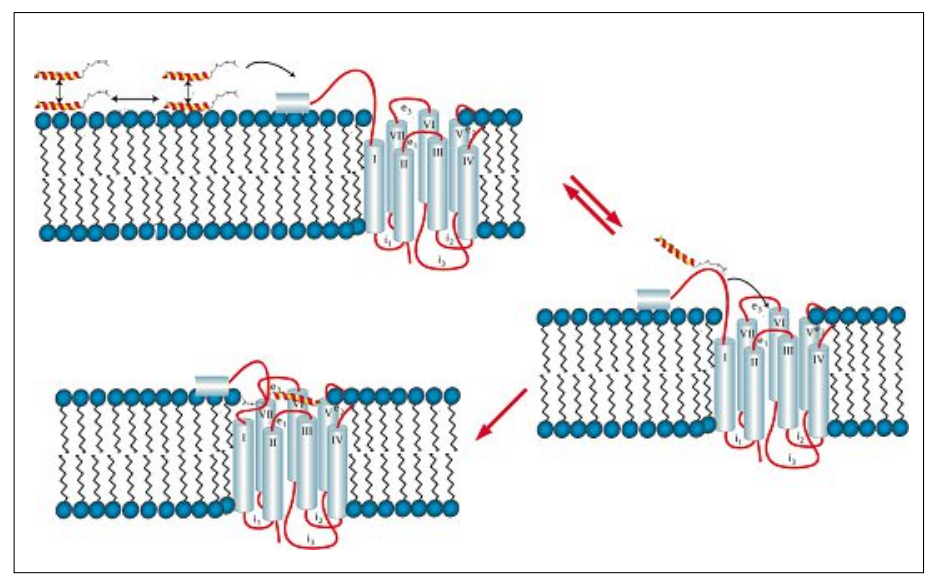

Fig. 2. Scheme describing the proposed events taking place during binding of PP to the $Y 4$ receptor. Following association on the membrane the ligand diffuses laterally. Once in vicinity of the receptor transient association to the $\mathrm{N}$-terminal domain might help to transfer it into the binding pocket of the receptor.

and a comparison with published NMR data of E. coli outer membrane protein A (an eight-stranded beta-barrel membrane protein) has revealed that the distances of loop anchoring points are comparable. The two approaches for gaining structural information on GPCRs are summarized in Fig. 1.

\section{Investigations of the Free $\mathrm{N}$-Terminal Domains of the Y4 Receptor}

The Y receptors contain 40-50 amino acid-long stretches at the $\mathrm{N}$-terminus in the extracellular space. ${ }^{[22]}$ To probe for their conformational preferences and their possible interactions with the ligands, which are peptide hormones of the NPY family, we have produced them in ${ }^{15} \mathrm{~N}$-labelled form. $\left[{ }^{16,17]} \quad\left[{ }^{15} \mathrm{~N},{ }^{1} \mathrm{H}\right]\right.$-HSQC spectra revealed them all to be largely unfolded with the exception of the $\mathrm{N}$-terminal domain from the Y4 receptor. ${ }^{[17]}$ This peptide contains two $\alpha$-helical stretches at the termini, separated by a long and flexible loop..$^{[16]}$ Interestingly, using surface-plasmon resonance (SPR), we could detect a weak ( $\mathrm{Kd}$ of approx. $50 \mu \mathrm{M}$ ) binding to the pancreatic polypeptide (PP), which is the peptide that associates with the Y4 receptor with the highest affinity. The interactions are largely mediated via electrostatic interactions, involving cationic residues from the
$\mathrm{N}$-terminal domain and anionic from the ligand PP. ${ }^{[16]}$ Considering that peptides from the NPY family associate with the membrane we speculate that a transient interaction of the peptides with the N-terminal domain might help to transfer them into the receptor binding-pocket (Fig. 2).

\section{Studies of 2-3 TM Helix Fragments from the Y4 Receptor}

As described above we have expressed the entire sequence of the Y4 receptor in the form of 2-3 TM fragments. The present status of the backbone assignments of residues of the $\mathrm{Y} 4$ receptor as derived from various fragments is summarized in Fig. 3. Since we studied the N-terminal domain of the Y4 receptor before, we decided to look at a construct which comprises this domain plus TM1 and TM2.[15] To be able to solubilize this protein we had to use a mixture of two detergents. Interestingly, the protein dramatically alters its solubility in the two components, dodecylphosphocholine (DPC) and 1-palmitoyl-2-hydroxysn-glycero-3-phospho-(1'-rac-glycerol) (LPPG) after purification by HPLC. A comparison of the $\mathrm{N}$-terminal domain quickly revealed that its motional properties are highly similar to those in the isolated $\mathrm{N}$-terminal domain, indicating that it is unlikely that this domain is fully folded 
in the entire receptor. A representative $\left[{ }^{15} \mathrm{~N},{ }^{1} \mathrm{H}\right]$-TROSY spectrum of NY4-TM1TM2 is depicted in Fig. 4. An initial analysis using chemical shifts indicated that the protein fragment is largely helically structured. In the meantime we have almost completely assigned the sidechains of this protein, and calculated the structure.[24] In that structure helices do not extend completely through the part predicted to be helical using a model of the Y4 receptor that is based on rhodopsin. Instead, the helices are interrupted by flexible hinges around polar or charged residues. Previously, we had looked at a similar construct from a yeast GPCR, the Ste $2 p$ receptor. In that fragment the content of polar residues in internal TM regions was much lower and secondary structure was present throughout the putatively helical regions. ${ }^{[18]}$ Moreover, we were able to observe tertiary contacts. We believe that the flexibility seen in Y4-TM1-TM2 is due to the high number of polar residues in TM1 or TM2 that prevents the helices from fully inserting into the micelle. ${ }^{[24]}$

A similar study using TM4-TM5 revealed that most resonances of TM4 were invisible in the proton-nitrogen correlation map, while signals due to TM5 and the loops could be rapidly assigned. Absence of signals from TM regions has been noticed by many others (for a review on NMR of membrane proteins see the excellent review of Kim et al.[25]). The examples of both sensory rhodopsin ${ }^{[5]}$ or proteorhodopsin,[6] for which almost all signals were found, indicates that this is not just a problem of size but rather related to exchange-broadening. A systematic study to investigate whether some of the missing signals could be rescued through exchange of polar residues in central locations of the TM helices did not reveal any improvements of spectra so far. In contrast, replacing Trp in the center of TM4 by Phe resulted in line-narrowing, at least for some residues. Expansions of $\left[{ }^{15} \mathrm{~N},{ }^{1} \mathrm{H}\right]-$ HSQC spectra of TM4-TM5 displaying a reporter signal that is easily recognized in the spectra (Gly159 from TM4) are depicted in Fig. 5. Obviously, the amide signal is exchange-broadened in the wild-type protein but significantly narrowed in the spectra from the W164F or W164A mutants. Whether mutations are successful in modifying the protein such that spectra of sufficient quality can be recorded is unclear at the moment. Similarly we worked on constructs comprising TM6-TM7 plus the C-terminal (cytosolic) 33 amino acid domain. Most of the resonances could be assigned with a few exceptions, this time mostly related to the repetitive sequence in some part and not to missing signals.

Chemical shift predictions with the program TALOS+${ }^{[26]}$ using the assigned

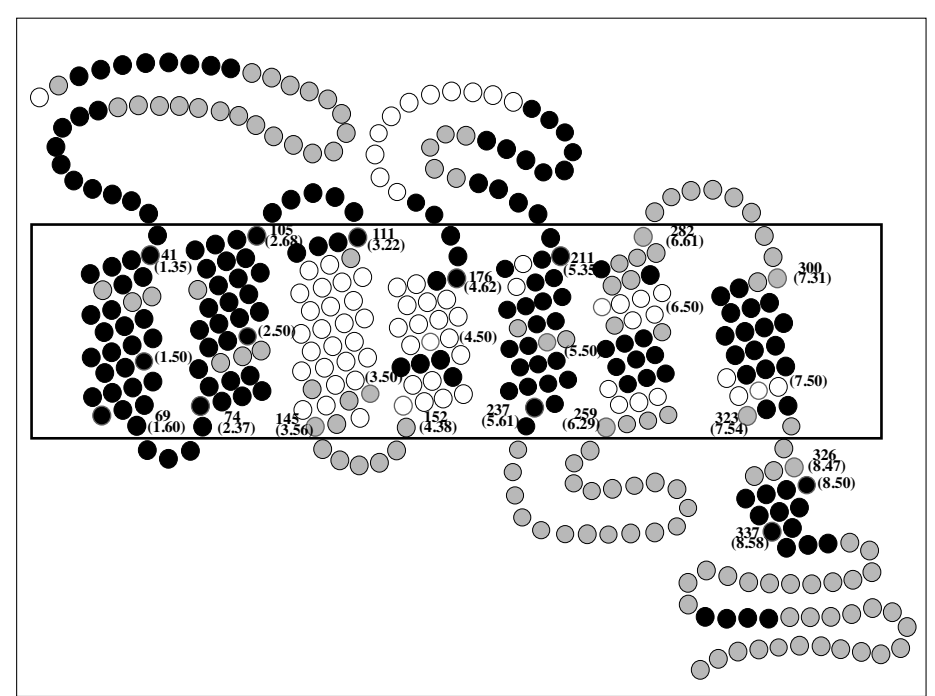

Fig. 3. Assignment status of fragments from the Y4 receptor. White spheres correspond to residues that could not be assigned yet, black and grey spheres to residues predicted to be helical or unstructured, respectively. Helix termini as well as the most-conserved positions are indicated by numbers, using the Ballesteros-Weinstein numbering. ${ }^{[23]}$

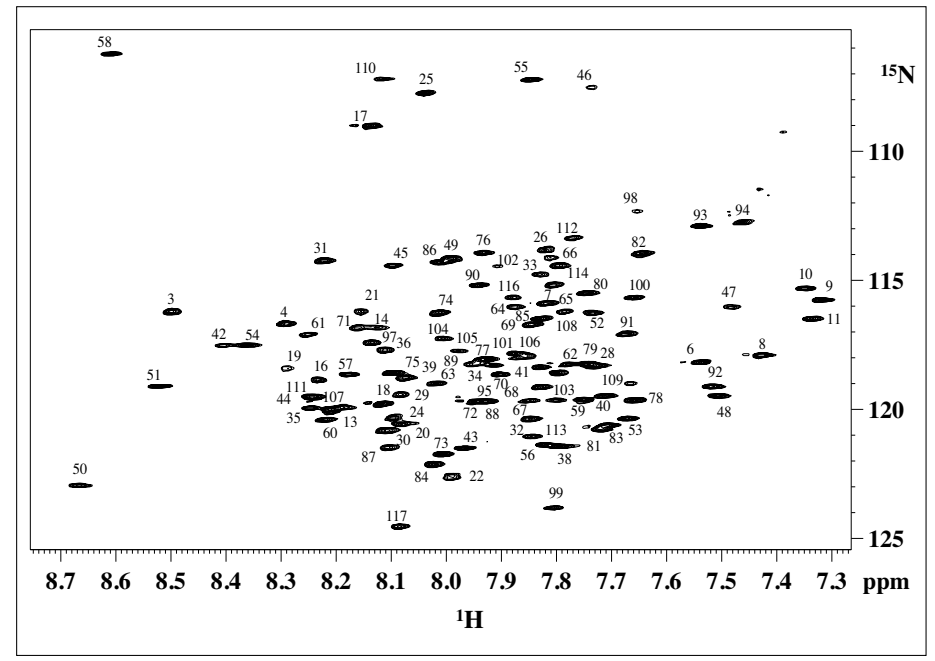

Fig. 4. $\left[{ }^{15} \mathrm{~N},{ }^{1} \mathrm{H}\right]$ TROSY spectrum of NY4 TM1-TM2 recorded at $700 \mathrm{MHz}$ and $320 \mathrm{~K}$ using a $500 \mu \mathrm{M}$ sample in $1 \%$ (28 mM) DPC, 6\% (118 mM) LPPG, 40 $\mathrm{mM}$ phosphate buffer, $20 \mathrm{mM}$ DTT, $\mathrm{pH}=6.0$. Peak numbering refers to the sequence location in the entire receptor.

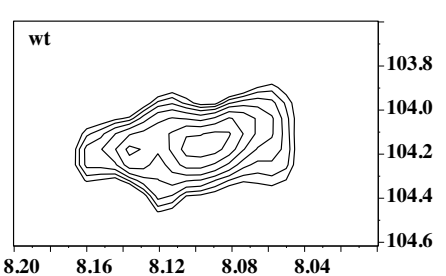

Fig. 5. Expansions of $\left[{ }^{15} \mathrm{~N},{ }^{1} \mathrm{H}\right]-\mathrm{HSQC}$ spectra from TM45 (top), S171C and S171A (middle) and W164F or W164A (bottom) displaying the cross peak for G159 that is part of TM4.
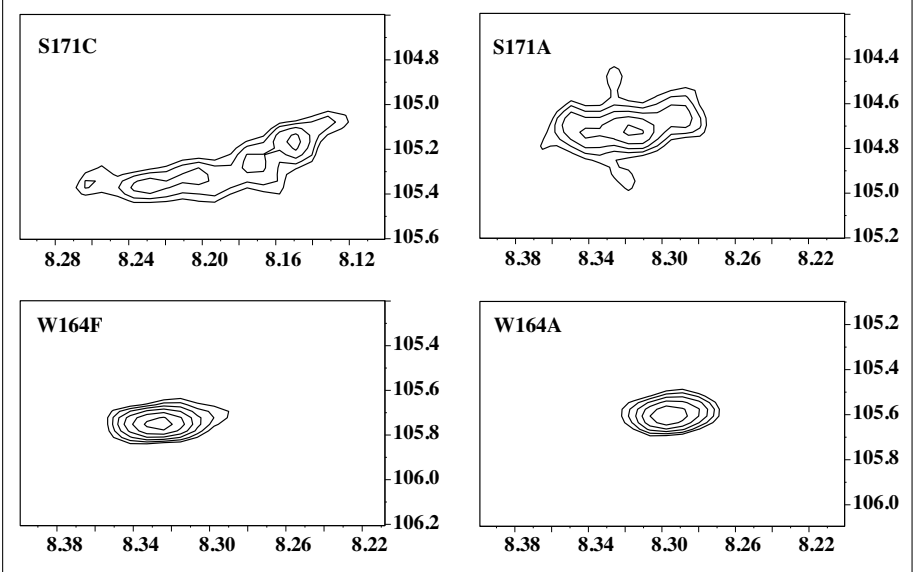

backbone chemical shifts revealed that the vast majority of residues from the TM regions are predicted to be helical (Fig. 3). In addition the putative helix in the cytosolic domain is detected, as well as a short amphiphatic helix prior to TM1. 


\section{Some Technical Remarks about the Work with Fragments}

We would like to add a few technical remarks here: First of all, the biochemistry of these fragments is far from trivial. While the entire receptors are certainly very difficult to produce in large quantities, [27] this is unfortunately also true at least to some extent for the fragments, although it is certainly easier. Purification to chemical homogeneity is another hurdle. Moreover, we have learned the hard way that careful characterization of these systems by sizeexclusion chromatography is important to recognize problems due to aggregation early on.

Backbone assignments of 2-TM or even larger constructs definitively require deuteration of the proteins, otherwise the sensitivity of the triple-resonance (TROSY-based) experiments is too low. Expression levels of the fragments in M9 (labeled) medium is mostly low, and protein production levels sometimes fall below detectable limits for cultures grown in deuterated water, as was the case for TM4-TM5. On that project we have used the fusion of TM4-TM5 to the N-terminal domain (NY4_TM4-TM5) to express the perdeuterated form of the protein, and later on adapted peaks in the ${ }^{15} \mathrm{~N}-\mathrm{TROSY}$ to the spectrum of TM4-TM5, using the ${ }^{15} \mathrm{~N}$-resolved NOESY to make sure that signals were correctly shifted.

Optimization of sample conditions is another tricky point. This mostly refers to the correct choice of detergent. Helical membrane proteins often comprise a delicate balance between protein-protein and protein-lipid contacts, and are often prone to aggregation and conformational exchange processes. The quality of spectra of a protein can be dramatically different in two detergents. ${ }^{[15,28]}$ We believe it is fair to say that the best detergent still needs to be determined empirically, a process reminiscent of the correct choice of buffer conditions in crystallization trials. A really frustrating experience in the last years was that samples that resulted in very promising spectra on ${ }^{15} \mathrm{~N}$-labeled protein in protonated detergents transformed into samples with limited stability and large propensities for aggregation when either the protein was deuterated or (often more detrimental) when perdeuterated detergents were used. Unfortunately, deuterated detergents are crucial when using ${ }^{13} \mathrm{C}$-resolved NOESY spectra because otherwise spectra will be dominated by detergent signals. A promising new medium for membrane proteins are the minidisks developed by Slighar and coworkers. ${ }^{[29]}$ While spectra for small membrane proteins suffer a lot from the increased transverse relaxation in these large entities (when compared to detergent mi- celles) for larger membrane proteins such as entire GPCRs this may not necessarily be the case any longer. Moreover, stability of protein has been claimed to be higher in minidisks allowing spectra to be recorded at higher temperatures and over more extended periods of time.

Fragments of GPCRs do not usually present rigidly folded membrane proteins with defined tertiary contacts. Moreover, they may not be uniquely anchored in the micelles. To characterize such inherently dynamical systems we have used a strategy that is based on measuring a couple of NMR parameters to characterize the systems: i) proton-proton NOEs as done traditionally for characterizing soluble proteins supplemented by secondary chemical shifts, ii) ${ }^{15} \mathrm{~N}\left\{{ }^{1} \mathrm{H}\right\}$-NOEs to independently detect flexibility, iii) micelle-integrating spin labels to probe for the topology of micelle integration, iv) proton-proton NOEs to detergent moieties or exchange peaks of amide protons to water. Both can be readily measured in the ${ }^{15} \mathrm{~N}$-resolved NOESY spectra, and provide further indirect evidence for micelle integration, v) RDC or
PREs to help establish tertiary contacts. We need to admit that we still have problems measuring RDCs in these systems with the required precision, and that we also have trouble with attaching the PRE probes selectively (our systems usually have multiple Cys sites, and we do not necessarily want to replace all of them).

\section{Grafting of Extracellular Loops of Y-Receptors onto beta-Barrel Scaffolds}

Replacing the fragile 7TM scaffold of GPCRs with an inherently more stable and synthetically more easily accessible scaffold is an interesting option for facilitating both biochemical and spectroscopic work on the study of the interaction of the GPCR extracellular loops and the receptors cognate ligands. So far we have focused in our studies in this field on the extracellular loops of the Y receptors. These have been proposed to present critical interaction sites with the neurohormone ligands. ${ }^{[30,31]}$ It is highly likely that the residues respon-
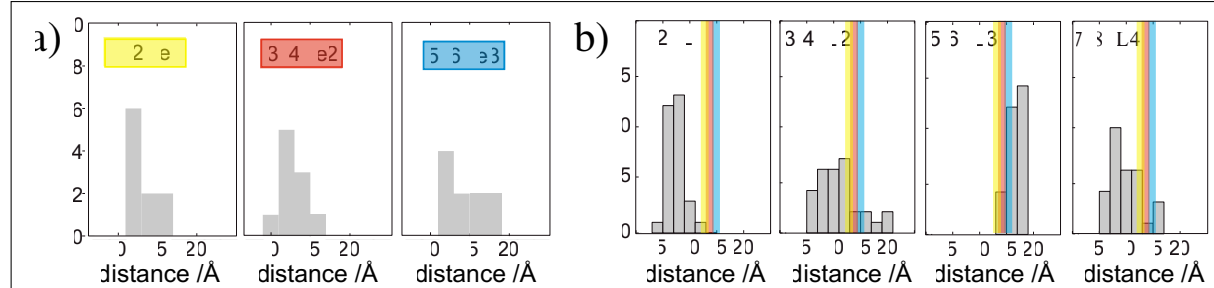

c)

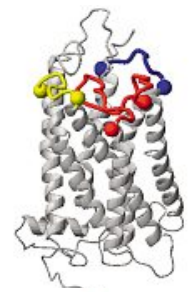

d)

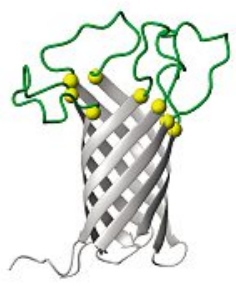

e)

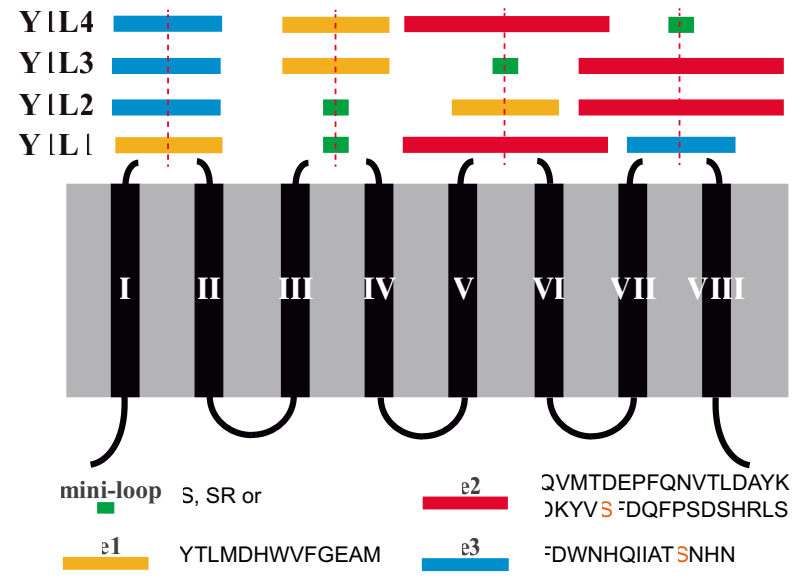

Fig. 6. Distance statistics observed in GPCRs and the OmpA scaffold and topology of the four expressed receptor constructs. a) Histograms of the distances between the anchor points for extracellular loops 1, 2, and 3 as found in a set of 10 GPCR crystal structures and b) for the extracellular loops in the NMR structures of two OmpA structures and one loop-shortened OmpA construct (10 conformers each). Average distances between the anchor points for the e1, e2, and e3 loop in the GPCR structures are indicated by yellow, red and blue bars, respectively. c) Ribbon representation of rhodopsin with the extracellular loops 1, 2, and 3 colored in yellow, red and blue, respectively. d) Ribbon representation of OmpA with the extracellular loops colored green and the loop anchor points shown in yellow. e) The four extracellular loops of OmpA were replaced with different arrangements of the three $\mathrm{Y} 1$ receptor extracellular loops. The fourth OmpA acceptor site was filled with a minimal turn-inducing sequence (called mini-loop here and depicted schematically in green). The extracellular loops 1, 2 and 3 of the $Y 1$ receptor are schematically represented by yellow, red and blue bars, respectively. At the bottom of the panel sequences of the grafted loops are depicted. Positions shown in red are cysteines in the natural receptor sequences and were replaced by Ser resiudes in our studies. 
sible for making interactions with the ligand need to have a suitable topological arrangement in order to meet the ligand's structural requirements. Under the assumption that the extracellular loops, which form the binding site for the ligand, are independent from each other a suitable scaffold must satisfy the following two requirements: i) The points in the scaffold to which foreign loops are attached (from now on called 'anchor points') should match the geometries observed in the native receptor and ii) the chemical environment of these anchor points should mimic the one found in the foreign receptor. The binding of many ligands to their cognate GPCRs in general[ ${ }^{32]}$ - and of the neurohormones of the NPY family in particular ${ }^{[33]}$ - has been proposed to be preceded by an initial generic binding to the membrane bilayer. In view of the membrane compartment theory the second requirement indicates that membrane-embedded scaffolds might represent more promising candidates as scaffolds. Due to the lack of high-resolution structural data on the $\mathrm{Y}$ receptors we instead extracted data on the orientation of extracellular loop anchor points from the published GPCR structures. An analysis of ten crystal structures of four different GPCRs reveals that the observed distances between loop attachment points are quite conserved (Fig. 6a).

The transmembrane domain of the outer membrane protein A (OmpA) from E. coli is a eight-stranded transmembrane beta-barrel protein ${ }^{[34]}$ which is structurally well-characterized. ${ }^{[35,36]}$ In addition it has been previously used as a scaffold. [37] Furthermore the distances between anchor-points in OmpA fall within the range as required by the GPCRs (Fig. 6b). While it is impossible to achieve a perfect match, it can be expected that mismatches may be partially compensated by structural adaptation, and if not, by inserting appropriate linkers between the grafted loops and the anchor point.

GPCRs possess three extracellular loops, while OmpA has four. Theoretically there are 24 different ways of arranging three donor loop sequences on the four acceptor sites of the scaffold. Considering that not all of these topologies are expedient it is possible to reduce this number by eliminating constructs with large distance mismatches or those in which the loops are placed in an erroneous sequential arrangement.

We have selected a set of four constructs (abbreviated as Y1L1, Y1L2, Y1L3, and Y1L4) in which the arrangement of the loops is the same as in the GPCRs, and which are characterized by a low overall distance mismatch. In theses constructs the three Y1-receptor extracellular loops (eY1) were grafted into different OmpA acceptor positions (Fig. 6e). In order to eliminate any possible interference of the residues of the fourth OmpA acceptor site with the grafted eY1, residues from the unused fourth $\beta$-barrel loop were replaced by a minimal turn-inducing motif of 1-2 amino acids that were previously shown to not interfere with the folding of the beta-barrel. ${ }^{[38]}$ These four constructs could all be expressed and purified as insoluble inclusion bodies from $E$. coli with high yields in the range of $100 \mathrm{mg}$ per liter of minimal medium culture. ${ }^{[19]}$ We could demonstrate that all proteins in which a single OmpA loop was replaced by an eY1 loop retained the $\beta$-barrel architecture. ${ }^{[20]}$ Indeed, we could also find suitable conditions for the refolding of the constructs carrying all three eY1 simultaneously. Non-denaturing SDSpage allowed for easy and rapid screening of a large number of refolding conditions on a small scale. Promising reactions were repeated on a larger scale, and the presence of a stable tertiary fold assessed by NMR. This allowed us to find conditions with $>75 \%$ refolding efficiencies for all four constructs. ${ }^{[19]}$

Because of the large size and the conformational flexibility in the OmpA scaffold ${ }^{39]}$ the quality of the NMR spectra from these constructs were not sufficient to allow detailed structural characterization. In order to study possible interactions of these constructs with NPY family neurohormones we thus resorted to chemical shift mapping using ${ }^{15} \mathrm{~N}$-labeled neurohormone peptides. We carried out titrations of the neurohormones with refolded unlabeled chimeric receptor constructs. Interestingly only small changes were seen in the neurohormones' spectra upon addition of two of the constructs (Y1L1 and Y1L2), whereas clear changes were observed for the other two constructs (Y1L3 and Y1L4) (Fig. 7a7c). Specifically, we observed a gradual reduction in peak intensity for resonances towards the C-terminus (Fig. 7d). We attribute this effect to slow exchange between the unbound and the bound state of the ligand, and to the fact that the bound state is either characterized by many different substates or further broadened through exchange. The fact that slow exchange between bound and unbound forms is present indicates that binding to the receptor constructs is likely in the low micromolar range. A more precise determination of the binding constant was unfortunately not yet possible, mainly due to complications resulting from the competing binding of the neurohormones to the detergent micelles into which the receptor constructs were reconstituted.

The observation that primarily the C-terminal residues of the neurohormones are involved in binding to the receptor's

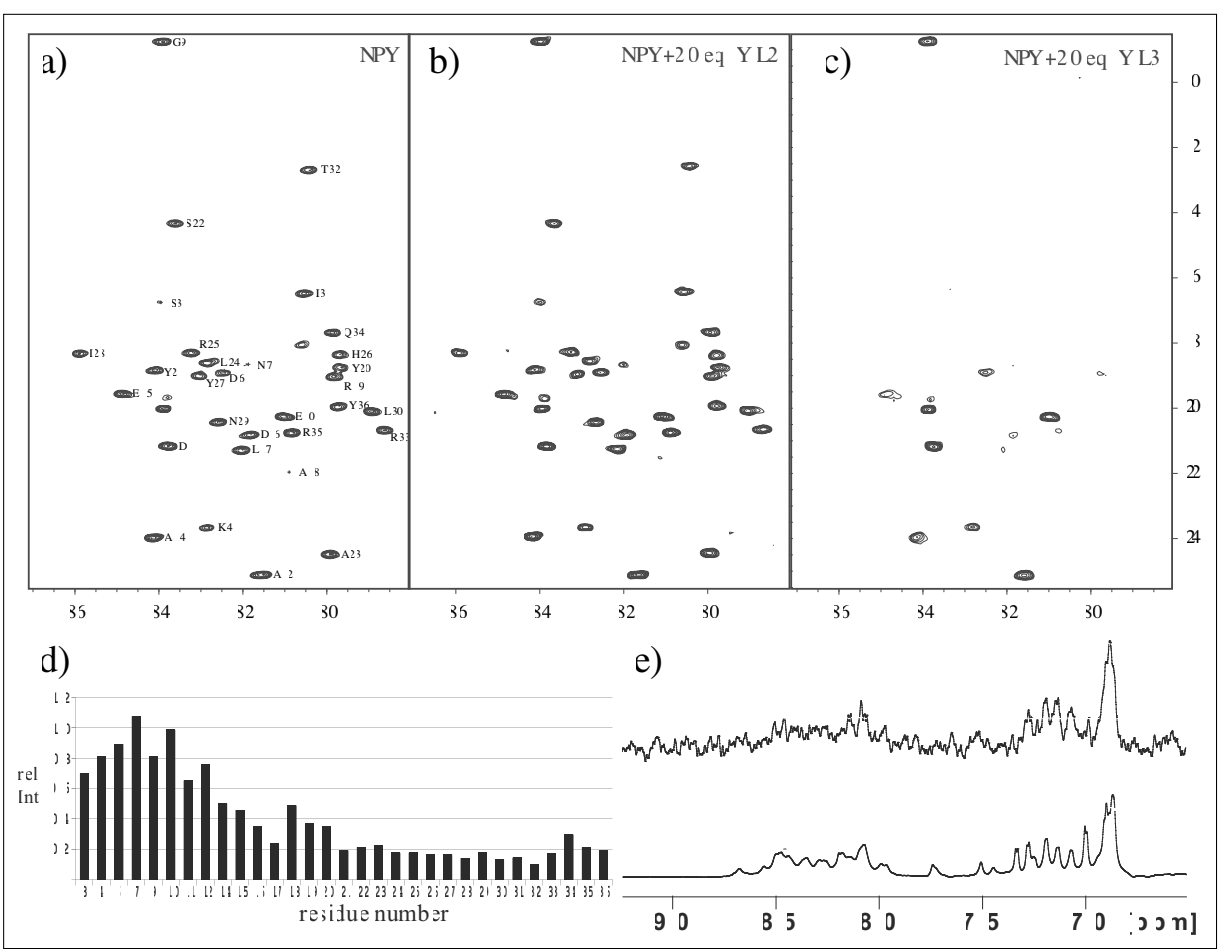

Fig. 7. Titration of ${ }^{15} \mathrm{~N}$-labeled porcine NPY (pNPY) with the four receptor constructs. To ${ }^{15} \mathrm{~N}$-labeled pNPY in $3 \%$ dihexanoylphosphatidylcholine (DHPC), $20 \mathrm{mM}$ sodium phosphate $\mathrm{pH}$ $6.5,100 \mathrm{mM} \mathrm{NaCl} 20$ equivalents of the indicated receptor constructs were added. a) The spectra for pNPY in the absence of receptor construct (top left), b) after addition of 20 eq. Y1L2 (top middle), and c) 20 eq. Y1L3 (top right). d) The decrease in peak intensities upon addition of 20 eq. Y1L3 to pNPY is mapped onto the pNPY sequence. e) STD spectrum of NPY in presence of 20 eq. Y1L3 (upper trace) as well as the reference proton spectrum (lower trace) displaying the region of amide and aromatic signals. 
extracellular loops is in agreement with data published by other groups ${ }^{[40]}$ and was further corroborated by us using saturation transfer difference ${ }^{41,42]}$ (STD) experiments ${ }^{[19]}$ (Fig. 7e).

The observed affinity for the receptor model is significantly weaker compared to the nanomolar dissociation constants observed between the neurohormones and the natural Y receptors. ${ }^{[43]}$ This may indicate that not all residues responsible for ligand binding were grafted to the scaffold, e.g. some residues from the N-terminal domain. We are presently investigating to which extent the $\mathrm{N}$-terminal domain can be introduced into the remaining (empty) acceptor loop of the scaffold. Alternatively, residues from the TM-domain might also contribute to binding. Including them in the scaffold is certainly much more challenging, and requires knowledge of their chemical nature and position to successfully place them inside the barrel. An alternative explanation is that the topology of the anchor points in the OmpA scaffold do not allow for an optimal positioning of the loops relative to each other. We tried to address this possibility by introducing flexible glycine-serine linkers in between the eY1 loops and the anchor points, but failed to observe increased affinity of these constructs towards the ligands.

\section{Conclusions}

In this paper we have reviewed our work aimed at obtaining structural data for G-protein coupled receptors by solution NMR methods. We have demonstrated that we can produce fragments covering the complete sequence of a human GPCR, the Y4 receptor. We have seen that chemical shift information from smaller fragments can be transferred onto longer constructs thereby facilitating the assignment process a lot. Whether the chemical shift information can also be transferred onto the entire receptor needs to be investigated in future. Of course, preparation of samples from the entire receptor that yield spectra of sufficient quality will be of prime importance. To successfully achieve this we will need to understand more about protein-lipid interactions and how to reconstitute the entire receptors in these systems. The grafting approach, about which we also reported in this paper, demonstrated the synthetic feasibility of such a receptor model. We are encouraged by the fact that the receptor model is recognized by the ligands. However, we need to admit that the binding affinity is much lower than for the wild-type receptor underlining the importance to closely mimic the topology of the binding site. We would like to conclude here that working on GPCR using solution NMR methods will remain a very challenging field, but we believe that we will witness important results in this field within the next five years.

\section{Acknowledgements}

We are indebted to the valuable contributions from coworkers of the Zerbe laboratory during the last decade. Coworkers on these projects were (in chronological order): Reto Bader, Mirjam Lerch-Bader, Alexey Neumoin, Chao Zou, Sowmini Kumaran, Harsha Kocherla, Xuan Shao, Jacopo Marino and Martin Poms. In addition, we are closely working together in a very fruitful collaboration with the labs of Fred Naider and Jeff M. Becker on a yeast GPCR, the Ste2p, where we are following similar approaches. Work on these projects has been funded by the Swiss National Science Foundation, the Forschungskredit der Universität Zürich and the OPO Foundation.

Received: August 2, 2012

[1] V. Katritch, V. Cherezov, R. C. Stevens, Trends Pharmacol. Sci. 2012, 33, 17 .

[2] K. Palczewski, T. Kumasaka, T. Hori, C. A. Behnke, H. Motoshima, B. A. Fox, I. Le Trong, D.C. Teller, T. Okada, R. E. Stenkamp, M. Yamamoto, M. Miyano, Science 2000, 289, 739.

[3] S. G. Rasmussen, B. T. DeVree, Y. Zou, A. C. Kruse, K. Y. Chung, T. S. Kobilka, F. S. Thian, P. S. Chae, E. Pardon, D. Calinski, J. M. Mathiesen, S. T. Shah, J. A. Lyons, M. Caffrey, S. H. Gellman, J. Steyaert, G. Skiniotis, W. I. Weis, R. K. Sunahara, B. K. Kobilka, Nature 2011, 477, 549

[4] A. Gautier, J. P. Kirkpatrick, D. Nietlispach, Angew. Chem. Int. Ed. Engl. 2008, 47, 7297.

[5] A. Gautier, H. R. Mott, M. J. Bostock, J. P. Kirkpatrick, D. Nietlispach, Nat. Struct. Mol. Biol. 2010, 17, 768

[6] S. Reckel, D. Gottstein, J. Stehle, F. Löhr, M. K. Verhoefen, M. Takeda, R. Silvers, M. Kainosho, C. Glaubitz, J. Wachtveitl, F. Bernhard, H. Schwalbe, P. Güntert, V. Dötsch, Angew. Chem. Int. Ed. Engl. 2011, 50, 11942.

[7] O. Zerbe, Angew. Chem. Int. Ed. Engl. 2012, 51, 860.

[8] S. Ahuja, E. Crocker, M. Eilers, V. Hornak, A. Hirshfeld, M. Ziliox, N. Syrett, P. J. Reeves, H. G. Khorana, M. Sheves, S. O. Smith, J. Biol. Chem. 2009, 284, 10190.

[9] J. L. Popot, D. M. Engelman, Annu. Rev. Biochem. 2000, 69, 881; J. L. Popot, D. M. Engelman, Biochemistry 1990, 29, 4031.

[10] T. Marti, J. Biol. Chem. 1998, 273, 9312.

[11] N. P. Martin, L. M. Leavitt, C. M. Sommers, M. E. Dumont, Biochemistry 1999, 38, 682.

[12] B. König, A. Arendt, J. H. McDowell, M. Kahlert, P. A. Hargrave, K. P. Hofmann, Proc. Natl. Acad. Sci. USA 1989, 86, 6878.

[13] P. L. Yeagle, J. L. Alderfer, A. D. Albert, Nat. Struct. Biol. 1995, 2, 832.

[14] H. Kocherla, J. Marino, X Shao, J. Graf, C. Zou, O. Zerbe, ChemBioChem 2012, 13, 818; A. Neumoin, B. Arshava, J. Becker, O. Zerbe, F. Naider, Biophys. J. 2007, 93, 467.
[15] C. Zou, F. Naider, O. Zerbe, J. Biomol. NMR 2008, 42, 257.

[16] C. Zou, S. Kumaran, S. Markovic, R. Walser, O. Zerbe, ChemBioChem 2008, 9, 2276.

[17] C. Zou, S. Kumaran, R. Walser, O. Zerbe, J. Pept. Sci. 2009, 15, 184.

[18] A. Neumoin, L. S. Cohen, B. Arshava, S. Tantry, J. M. Becker, O. Zerbe, F. Naider, Biophys. J. 2009, 96, 3187.

[19] R. Walser, J. H. Kleinschmidt, O. Zerbe, ChemBioChem 2011, 12, 1690.

[20] R. Walser, J. H. Kleinschmidt, A. Skerra, O. Zerbe, in press.

[21] P. L. Yeagle, A. D. Albert, Methods Enzymol. 2002, 343, 223; D. F. Mierke, L. Mao, M. Pellegrini, A. Piserchio, J. Plati, N. Tsomaia, Biochem Soc Trans 2007, 35, 721; M. Pellegrini, D. F. Mierke, Biopolymers 1999, 51, 208.

[22] D. Larhammar, E. Salaneck, Neuropeptides 2004, 38, 141.

[23] J. Ballesteros, H. Weinstein, Methods. Neurosci. $1995,25,366$.

[24] X. Shao, C. Zou, F. Naider, O. Zerbe, Biophys. J. 2012, 103, 817

[25] H. J. Kim, S. C. Howell, W. D. Van Horn, Y. H. Jeon, C. R. Sanders, Prog. Nucl. Magn. Reson. Spectrosc. 2009, 55, 335.

[26] Y. Shen, F. Delaglio, G. Cornilescu, A. Bax, J. Biomol. NMR 2009, 44, 213.

[27] V. Sarramegna, I. Muller, A. Milon, F. Talmont, Cell Mol. Life Sci. 2006, 63, 1149.

[28] R. D. Krüger-Koplin, P. L. Sorgen, S. T. KrügerKoplin, I. O. Rivera-Torres, S. M. Cahill, D. B. Hicks, L. Grinius, T. A. Krulwich, M. E. Girvin, J. Biomol. NMR 2004, 28, 43.

[29] A. Nath, W. M. Atkins, S. G. Sligar, Biochemistry 2007, 46, 2059; E. N. Lyukmanova, Z. O. Shenkarev, A. S. Paramonov, A. G. Sobol, T. V. Ovchinnikova, V. V. Chupin, M. P. Kirpichnikov, M. J. Blommers, A. S. Arseniev, J. Am. Chem. Soc. 2008, 130, 2140.

[30] N. Merten, D. Lindner, N. Rabe, H. Rompler, K. Morl, T. Schoneberg, A. G. Beck-Sickinger, J. Biol. Chem. 2007, 282, 7543 .

[31] C. Robin-Jagerschmidt, I. Sylte, C. Bihoreau, L. Hendricksen, A. Calvet, S. G. Dahl, C. Benicourt, Mol. Cell. Endocrinol. 1998, 139, 187; P. Walker, M. Munoz, R. Martinez, M. C. Peitsch, J. Biol. Chem. 1994, 269, 2863.

[32] D. F. Sargent, R. Schwyzer, Proc. Natl. Acad. Sci. USA 1986, 83, 5774.

[33] R. Bader, O. Zerbe, ChemBioChem 2005, 6, 1520.

[34] N. Ruiz, D. Kahne, T. J. Silhavy, Nat Rev Microbiol. 2006, 4, 57.

[35] A. Arora, F. Abildgaard, J. H. Bushweller, L. K. Tamm, Nat. Struct. Biol. 2001, 8, 334.

[36] A. Pautsch, G. E. Schulz, Nat. Struct. Biol. 1998, 5, 1013.

[37] M. U. Johansson, S. Alioth, K. Hu, R. Walser, R. Koebnik, K. Pervushin, Biochemistry 2007, 46,1128 .

[38] R. Koebnik, J. Mol. Biol. 1999, 285, 1801.

[39] C. Fernandez, K. Adeishvili, K. Wüthrich, Proc. Natl. Acad. Sci. USA 2001, 98, 2358.

[40] A. G. Beck-Sickinger, H. A. Wieland, H. Wittneben, K. D. Willim, K. Rudolf, G. Jung, Eur. J. Biochem. 1994, 225, 947.

[41] M. Mayer, B. Meyer, Angew. Chem. Intl. Ed. 1999, 38,1784

[42] M. Mayer, B. Meyer, J. Am. Chem. Soc. 2001, 123,6108 .

[43] D. Larhammar, Regul. Pept. 1996, 62, 1. 